\title{
Global well-posedness of a class of stochastic equations with jumps
}

Guoli Zhou*

"Correspondence:

zhouguoli736@126.com

School of Mathematics and

Statistics, Chong Qing University,

Chong Qing, P.R. China

\begin{abstract}
The existence and uniqueness of a mild solution to stochastic equations with jumps are established, a stochastic Fubini theorem and a type of Burkholder-Davis-Gundy inequality are proved, and the two formulas are used to study the regularity property of the mild solution of a general stochastic evolution equation perturbed by jump processes. As applications, the regularity of a stochastic heat equation with jump is given.

MSC: $76 \mathrm{~S} 05 ; 60 \mathrm{H} 15$
\end{abstract}

Keywords: stochastic evolution equation; Lévy processes; mild solution

\section{Introduction}

In recent years, there have been many monographs concerning stochastic partial differential equations with Lévy jump and their applications in physics, economics, statistical mechanics, fluid dynamics and finance etc. For this theory and its applications, one can see [1-3] and references therein. In this article, the existence, uniqueness, regularity for the mild solution of stochastic partial differential equations with Lévy jump are studied. There are a lot of works dealing with existence and uniqueness for stochastic partial differential equations with jump processes. In [4], the existence and uniqueness for solutions of stochastic reaction diffusion equations driven by Poisson random measures are obtained. In [5], Malliavin calculus is applied to study the absolute continuity of the law of the solutions of stochastic reaction diffusion equations driven by Poisson random measures. In [6], a minimal solution is obtained for the stochastic heat equation driven by non-negative Lévy noise with coefficients of polynomial growth. In [7], a weak solution is established for the stochastic heat equation driven by stable noise with coefficients of polynomial growth. In [8], the existence and uniqueness for solutions of stochastic generalized porous media equations with Lévy jump are obtained. In [9], the strong solutions to a large class of stochastic equations with Lévy noise are obtained in variational framework. And it is shown in [9] that the results can be applied to stochastic reaction-diffusion equations, Burgers-type equations, 2D Navier-Stokes equations, $p$-Laplace equations and porous media equations with locally monotone perturbations.

The main aim of this paper is to study the existence, uniqueness and regularity of the stochastic equation

$$
d X(t)=[A(X(t))+B(X(t))] d t+Q(X(t)) d W(t)+\int_{Z} F(X(t-), x) \tilde{N}(d t, d x) .
$$

(0) 2013 Zhou; licensee Springer. This is an Open Access article distributed under the terms of the Creative Commons Attribution License (http://creativecommons.org/licenses/by/2.0), which permits unrestricted use, distribution, and reproduction in any medium, provided the original work is properly cited. 
In [10], the intensity measure $\lambda$ of $\widetilde{N}(d t, d x)$ is finite, while the intensity measure in this article is $\sigma$-finite, and also the classical Lipschitz condition (2.5) in [10] is relaxed to condition (A.5) in this article. The author of this article proves the existence and uniqueness of a mild solution of (1.1), the continuity of the solution with respect to initial data. And then a stochastic Fubini theorem is established for the compensated Poisson random measure whose intensity measure is $\sigma$-finite compared to a finite case in [10]. Furthermore, a new type of the Burkholder-Davis-Gundy inequality, which is more precise than Lemma 2.2 in [10], is gotten. The two formulas are basic in stochastic analysis. Using the formulas, the author gets the regularity property of a mild solution of (1.1) without conditions (2.15) and (2.16) in [10] which are critical there.

The article is organized as follows. In Section 2, we present the framework. Existence, uniqueness and regularity are proved in Section 3. In Section 4, two examples including stochastic heat equations with Lévy jump are given.

\section{Some preliminaries}

Let $\left(U,\|\|_{U}\right),(H,\|\|)$ be separable Hilbert spaces and $Z$ be a Banach space. Let $L_{2}(U, H)$ denote the space of all Hilbert-Schmidt operators from $U$ to $H$, and set \|\|$_{L_{2}}:=\|\|_{L_{2}(U, H) \text {. Let }}$ $W(t), t \geq 0$ be a cylindrical Wiener process in $U$ on a probability space $(\Omega, \mathcal{F}, P)$ with a normal filtration $\mathcal{F}_{t}, t \geq 0$ and let $N(d t, d z)$ be a Poisson random measure associated with the compensated $\left(\mathcal{F}_{t}\right)$-martingale measure defined as $\tilde{N}(d t, d z):=N(d t, d z)-\lambda(d z) d t$, where $\lambda(d z)$ defined on a measurable space $Z$ is the intensity measure of $N(d t, d z)$. In this article we study the following equation:

$$
\begin{aligned}
d X(t)= & {[A X(t)+B(X(t))] d t+Q(X(t)) d W(t) } \\
& +\int_{Z} F(X(t-), z) \widetilde{N}(d t, d z), \quad t \in[0, T]
\end{aligned}
$$

with initial condition $X(0)=\eta$, and the coefficients in equation (2.1) satisfy the following conditions:

(A.1) $A: D(A) \rightarrow H$ is the infinitesimal generator of a $C_{0}$-semigroup $S(t), t \geq 0$.

(A.2) $B: H \rightarrow H$ is $\mathcal{B}(H) / \mathcal{B}(H)$-measurable, and there exists a positive constant $C$ satisfying

$$
\|B(x)-B(y)\| \leq C\|x-y\| \quad \text { for all } x, y \in H .
$$

(A.3) $Q: H \rightarrow L(U, H)$ is strongly continuous, i.e. the mapping

$$
x \rightarrow Q(x) u
$$

is continuous from $H$ to $H$ for each $u \in U$.

(A.4) For all $t \in] 0, T]$ and $x \in H$, we have

$$
S(t) Q(x) \in L_{2}(U, H) .
$$

(A.5) There is a square integrable mapping $M:[0,+\infty[\rightarrow[0, \infty[$ such that

$$
\|S(t)(Q(x)-Q(y))\|_{L_{2}} \leq M(t)\|x-y\|
$$


and

$$
\|S(t) Q(x)\|_{L_{2}} \leq M(t)(1+\|x\|)
$$

for all $t \in] 0, T]$ and $x, y \in H$.

(A.6) There is a positive constant $C$ like (A.2) such that

$$
\int_{Z}\|F(x, z)-F(y, z)\|^{2} \lambda(d z) \leq C\|x-y\|^{2}
$$

and

$$
\int_{Z}\|F(x, z)\|^{2} \lambda(d z) \leq C\left(1+\|x\|^{2}\right),
$$

where $F: H \times Z \rightarrow H$ is $\mathcal{B}(H) \times \mathcal{B}(Z) / \mathcal{B}(H)$ measurable.

For fixed $T>0$, we define:

$$
\begin{aligned}
\mathcal{H}^{2}(T, H):= & \{H \text { valued predictable process } X \text { with } \\
& \left.\|X\|_{H^{2}}:=\sup _{t \in[0, T]}\left(E\left(\|X(t)\|^{2}\right)\right)^{1 / 2}<\infty\right\} .
\end{aligned}
$$

Obviously, $\left(\mathcal{H}^{2}(T, H),\|\|_{H^{2}}\right)$ is a Banach space. For technical reasons we define \|\|$_{2, \lambda, T}$, $\lambda \geq 0$ on $\mathcal{H}^{2}(T, H)$ as

$$
\|X\|_{2, \lambda, T}:=\sup _{t \in[0, T]} e^{-\lambda t}\left(E\left(\|X(t)\|^{2}\right)\right)^{1 / 2} .
$$

Then, for $X \in \mathcal{H}^{2}(T, H)$ and $\lambda \geq 0$, we have

$$
\|X\|_{2, \lambda, T} \leq\|X\|_{H^{2}} \leq e^{\lambda T}\|X\|_{2, \lambda, T}
$$

For the reader's convenience, before giving our main results, we cite some theorems here which will be needed later.

Contraction theorem (i) Let $\left(E,\|\|_{E}\right)$ and $\left(\Lambda,\|\|_{\Lambda}\right)$ be two Banach spaces. Let the mapping $G: \Lambda \times E \rightarrow$ E satisfy

$$
\|G(\lambda, x)-G(\lambda, y)\|_{E} \leq \alpha\|x-y\|_{E}, \quad \alpha \in[0,1[
$$

for all $\lambda \in \Lambda$ and $x, y \in E$. Then there exists exactly one mapping $\varphi: \Lambda \rightarrow E$ such that

$$
\varphi(\lambda)=G(\lambda, \varphi(\lambda))
$$

for all $\lambda \in \Lambda$.

(ii) If we assume in addition that the mapping $\lambda \rightarrow G(\lambda, x)$ is continuous from $\Lambda$ to $E$ for all $x \in E$, we get that $\varphi: \Lambda \rightarrow E$ is continuous. 
(iii) If the mapping $\lambda \rightarrow G(\lambda, x)$ is not only continuous from $\Lambda$ to $E$ for all $x \in E$, but there even exists an $L \geq 0$ such that

$$
\|G(\lambda, x)-G(\tilde{\lambda}, x)\|_{E} \leq L\|\lambda-\tilde{\lambda}\|_{\Lambda}
$$

for all $x \in E$, then the mapping $\varphi: \Lambda \rightarrow E$ is Lipschitz continuous.

Theorem [11] If a process $\Psi$ is adapted to $\mathcal{F}_{t}, t \in[0, T]$, and stochastically continuous with values in a Banach space $E$, then there exists a predictable version of $\Psi$.

Definition 2.1 An $H$-valued predictable process $X(t), t \in[0, T]$ is called a mild solution of (2.1) if

$$
\begin{aligned}
X(t)= & S(t) \eta+\int_{0}^{t} S(t-s) B(X(s)) d s+\int_{0}^{t} S(t-s) Q(X(s)) d W(s) \\
& +\int_{0}^{t} \int_{Z} S(t-s) F(X(s-), z) \tilde{N}(d s, d z)
\end{aligned}
$$

for each $t \in[0, T]$.

\section{Existence, uniqueness and regularity}

Theorem 3.1 Assume that conditions from (A.1) to (A.6) hold, then there exists a unique mild solution $X(\eta) \in \mathcal{H}^{2}(T, H)$ of $(2.1)$ with the initial condition

$$
\eta \in L^{2}\left(\Omega, \mathcal{F}_{0} ; H\right)=: L_{0}^{2}
$$

In addition we get that the mapping

$$
\begin{aligned}
& X: L_{0}^{2} \rightarrow \mathcal{H}^{2}(T, H), \\
& \eta \rightarrow X(\eta)
\end{aligned}
$$

is Lipschitz continuous.

Proof Fix $t \in[0, T], \eta \in L_{0}^{2}$ and $X \in \mathcal{H}^{2}(T, H)$, and define

$$
\begin{aligned}
\mathcal{F}(\eta, X)(t):= & S(t) \eta+\int_{0}^{t} S(t-s) B(X(s)) d s+\int_{0}^{t} S(t-s) Q(X(s)) d W(s) \\
& +\int_{0}^{t} S(t-s) F(X(s-), z) \tilde{N}(d s, d z) .
\end{aligned}
$$

In the following two steps, it comes to prove that

$$
\mathcal{F}: L_{0}^{2} \times \mathcal{H}^{2}(T, H) \rightarrow \mathcal{H}^{2}(T, H)
$$

The first step: It is proved that the mapping $\mathcal{F}$ is well defined.

1. Let $h \in H$, as

$$
\left\langle 1_{[0, t]}(s) S(t-s) B(X(s)), h\right\rangle_{H}=\left\langle B(X(s)), 1_{[0, t]}(s) S^{*}(t-s) h\right\rangle_{H} .
$$


By the strongly continuity of $S(t)$, we know $1_{[0, t]}(s) S(t-s) B(X(s))$ is predictable. And together with (A.2), we know the Bochner integral $\int_{0}^{t} S(t-s) B(X(s)) d s, t \in[0, T]$, is well defined.

2. Let $\left\{e_{i}\right\}$ and $\left\{h_{i}\right\}$ be an orthonormal basis respectively of $U$ and $H$. Because

$$
\left\langle h_{i}, 1_{[0, t]}(s) S(t-s) Q(X(s)) e_{j}\right\rangle_{H}
$$

is predictable, and by (A.5), it is easy to see $1_{[0, t]}(s) S(t-s) Q(X(s)) \in \mathcal{N}_{W}^{2}(0, T)$. So, the stochastic integral $\int_{0}^{t} S(t-s) Q(X(s)) d W(s)$ is well defined.

3. Similarly to 1 , it is easy to see that $1_{[0, t]}(s) S(t-s) F(X(s), z)$ is predictable, and by (A.6) we have

$$
E\left\|\int_{0}^{t} \int_{Z} S(t-s) F(X(s-), z) \tilde{N}(d s, d z)\right\|^{2}<+\infty
$$

Therefore the stochastic integral $\int_{0}^{t} \int_{Z} S(t-s) F(X(s-), z) \tilde{N}(d s, d z)$ is well defined.

The second step: We prove that $\mathcal{F}(\eta, X) \in \mathcal{H}^{2}(T, H)$ for all $\eta \in L_{0}^{2}$ and $X \in \mathcal{H}^{2}(T, H)$.

1. Obviously $S(t) \eta, t \in[0, T]$, is an element of $\mathcal{H}^{2}(T, H)$.

2. There is a version of the second summand $\int_{0}^{t} S(t-s) B(X(s)) d s, t \in[0, T]$, which is an element of $\mathcal{H}^{2}(T, H)$.

Similarly to the argument of the first step, we know $\int_{0}^{t} S(t-s) B(X(s)) d s$ is adapted for $\left(\mathcal{F}_{t}\right)_{t \geq 0}$. Then we will show $\int_{0}^{t} S(t-s) B(X(s)) d s$ is continuous a.s. Let $\left.\left.0 \leq t<t_{0}, r \in\right] 1,2\right]$. By (A.2), we have

$$
\begin{aligned}
& \left\|\int_{0}^{t} S(t-s) B(X(s)) d s-\int_{0}^{t_{0}} S\left(t_{0}-s\right) B(X(s)) d s\right\| \\
& \leq\left\|\int_{0}^{t}\left(S(t-s)-S\left(t_{0}-s\right)\right) B(X(s)) d s\right\|+\left\|\int_{t}^{t_{0}} S\left(t_{0}-s\right) B(X(s)) d s\right\| \\
& \leq\left\|\int_{0}^{t / r}\left(S(t-r s)-S\left(t_{0}-r s\right)\right) S((r-1) s) B(X(s)) d s\right\|+\left\|\int_{t / r}^{t} S(t-s) B(X(s)) d s\right\| \\
& \quad+\left\|\int_{t / r}^{t} S\left(t_{0}-s\right) B(X(s)) d s\right\|+\left\|\int_{t}^{t_{0}} S\left(t_{0}-s\right) B(X(s)) d s\right\| \\
& \leq\left\|\int_{0}^{t / r}\left(S(t-r s)-S\left(t_{0}-r s\right)\right) S((r-1) s) B(X(s)) d s\right\|+\bar{C} M(T) \int_{t / r}^{t}(1+\|X(s)\|) d s \\
& \quad+\bar{C} M(T) \int_{t / r}^{t}(1+\|X(s)\|) d s+\bar{C} M(T) \int_{t}^{t_{0}}(1+\|X(s)\|) d s \\
& \leq \int_{0}^{t / r}\left\|\left(S(t-r s)-S\left(t_{0}-r s\right)\right) S((r-1) s) B(X(s))\right\| d s \\
& \quad+4 \bar{C} M(T)(T)^{\frac{1}{2}}\left(\int_{t / r}^{t}\left(1+\|X(s)\|^{2}\right) d s\right)^{1 / 2} \\
& \quad+2 \bar{C} M(T)(T)^{\frac{1}{2}}\left(\int_{t}^{t_{0}}\left(1+\|X(s)\|^{2}\right) d s\right)^{1 / 2} .
\end{aligned}
$$


In the above inequalities, we set $M_{T}:=\sup _{t \in[0, T]}\|S(t)\|_{L(H)}$ and $\bar{C}:=\max \{C,\|B(0)\|\}$, where $C$ is as in (A.2). As we can derive that

$$
\begin{aligned}
\int_{0}^{t / r}\|S((r-1) s) B(X(s))\| d s & \leq \bar{C} M(T) \int_{0}^{t / r}(1+\|X(s)\|) d s \\
& \leq \bar{C} M(T)\left(\int_{0}^{t} 2\left(1+\|X(s)\|^{2}\right) d s\right)^{1 / 2}<+\infty .
\end{aligned}
$$

Letting $t \rightarrow t_{0}$, and then $r \rightarrow 1$, by (A.2), strong continuity of $S(t)$ and dominated convergence theorem, we know, for all most $w \in \Omega$, we have

$$
\int_{0}^{t} S(t-s) B(X(s)) d s \rightarrow \int_{0}^{t_{0}} S\left(t_{0}-s\right) B(X(s)) d s \quad \text { for all } 0 \leq t<t_{0} \leq T .
$$

Similarly we can get the same conclusion for $0 \leq t_{0}<t \leq T$ when $t \downarrow t_{0}$, so we have proved $\int_{0}^{t} S(t-s) B(X(s)) d s$ is continuous a.s. for $t \in[0, T]$.

As we have

$$
\begin{aligned}
E\left(\left\|\int_{0}^{t} S(t-s) B(X(s)) d s\right\|^{2}\right) & \leq E \int_{0}^{t}\left(\|S(t-s) B(X(s))\|^{2}\right) d s T \\
& \leq(2 T)(\bar{C} M(T))^{2} E \int_{0}^{t}\left(1+\|X(s)\|^{2}\right) d s \\
& \leq 2(T \bar{C} M(T))^{2}\left(1+\|X\|_{H^{2}}^{2}\right) .
\end{aligned}
$$

Therefore we have proved $\int_{0}^{t} S(t-s) B(X(s)) d s \in \mathcal{H}^{2}(T, H)$.

3. There is a version of $\int_{0}^{t} S(t-s) Q(X(s)) d W(s), t \in[0, T]$, which is in $\mathcal{H}^{2}(T, H)$.

First we fix $r \in] 1,2]$, then

$$
\int_{0}^{t / r} S(t-s) Q(X(s)) d W(s)=\int_{0}^{t / r} S(t-r s) S((r-1) s) Q(X(s)) d W(s) .
$$

Let us define

$$
\Phi^{r}:=1_{[0, T]}(s) S((r-1) s) Q(X(s)) .
$$

Then it is clear that $\Phi^{r} \in \mathcal{N}_{W}^{2}(0, T)$. By (A.2), we have

$$
\begin{aligned}
E \int_{0}^{T}\left\|\Phi^{r}(s)\right\|_{L_{2}}^{2} d s & =E \int_{0}^{T}\left\|1_{[0, T]}(s) S((r-1) s) Q(X(s))\right\|_{L_{2}}^{2} d s \\
& \leq E \int_{0}^{T} 2(M((r-1) s))^{2}\left(1+\|X(s)\|^{2}\right) d s \\
& \leq \int_{0}^{T} 2(M((r-1) s))^{2} d s\left(1+\sup _{t \in[0, T]} E\left(\|X(t)\|^{2}\right)\right) \\
& \leq \frac{1}{r-1} \int_{0}^{(r-1) T} 2(M(s))^{2} d s\left(1+\sup _{t \in[0, T]} E\left(\|X(t)\|^{2}\right)\right) .
\end{aligned}
$$


Similarly we have $1_{[0, t]}(s) S(t-s) Q(X(s)) \in \mathcal{N}_{W}^{2}(0, T)$ and $\int_{0}^{t} S(t-s) Q(X(s)) d W(s)$ is $\mathcal{F}_{t^{-}}$ measurable. Next we are going to show

$$
t \rightarrow \int_{0}^{t} S(t-s) Q(X(s)) d W(s)
$$

is continuous in the mean square and therefore stochastically continuous. Indeed, let $0 \leq$ $t<t_{0} \leq T$, we get

$$
\begin{aligned}
& E\left(\left\|\int_{0}^{t} S(t-s) Q(X(s)) d W(s)-\int_{0}^{t_{0}} S\left(t_{0}-s\right) Q(X(s)) d W(s)\right\|^{2}\right) \\
& \leq 2 E\left(\left\|\int_{0}^{t}\left(S\left(t_{0}-s\right)-S(t-s)\right) Q(X(s)) d W(s)\right\|^{2}\right) \\
& +2 E\left(\left\|\int_{t}^{t_{0}} S\left(t_{0}-s\right) Q(X(s)) d W(s)\right\|^{2}\right) \\
& =2 E \int_{0}^{t}\left\|\left(S\left(t_{0}-s\right)-S(t-s)\right) Q(X(s))\right\|_{L_{2}}^{2} d s+2 E \int_{t}^{t_{0}}\left\|S\left(t_{0}-s\right) Q(X(s))\right\|_{L_{2}}^{2} d s \\
& =2 E \int_{0}^{t / r}\left\|\left(S\left(t_{0}-r s\right)-S(t-r s)\right) \Phi^{r}(s)\right\|_{L_{2}}^{2} d s \\
& +2 E \int_{t / r}^{t}\left\|\left(S\left(t_{0}-s\right)-S(t-s)\right) Q(X(s))\right\|_{L_{2}}^{2} d s \\
& +2 E \int_{t}^{t_{0}}\left\|S\left(t_{0}-s\right) Q(X(s))\right\|_{L_{2}}^{2} d s \\
& \leq 2 E \int_{0}^{t / r}\left\|\left(S\left(t_{0}-r s\right)-S(t-r s)\right) \Phi^{r}(s)\right\|_{L_{2}}^{2} d s+4 E \int_{t / r}^{t}\left\|\left(S\left(t_{0}-s\right)\right) Q(X(s))\right\|_{L_{2}}^{2} d s \\
& +4 E \int_{t / r}^{t}\|(S(t-s)) Q(X(s))\|_{L_{2}}^{2} d s+2 E \int_{t}^{t_{0}}\left\|S\left(t_{0}-s\right) Q(X(s))\right\|_{L_{2}}^{2} d s \\
& \leq 2 E\left(\int_{0}^{t / r} \sum_{i}\left\|\left(S\left(t_{0}-r s\right)-S(t-r s)\right) \Phi^{r}(s) e_{i}\right\|^{2} d s\right) \\
& +8 E \int_{t / r}^{t} M^{2}\left(t_{0}-s\right)\left(1+\|X(s)\|^{2}\right) d s \\
& +8 E \int_{t / r}^{t} M^{2}(t-s)\left(1+\|X(s)\|^{2}\right) d s+4 E \int_{t}^{t_{0}} M^{2}\left(t_{0}-s\right)\left(1+\|X(s)\|^{2}\right) d s \\
& =2 E\left(\int_{0}^{t / r} \sum_{i}\left\|\left(S\left(t_{0}-r s\right)-S(t-r s)\right) \Phi^{r}(s) e_{i}\right\|^{2} d s\right) \\
& +8\left[1+\|X\|_{H^{2}}^{2}\right]\left(\int_{t_{0}-t}^{t_{0}-t / r} M^{2}(s) d s+\int_{0}^{t(r-1) / r} M^{2}(s) d s\right) \\
& +4 E \int_{0}^{t_{0}-t} M^{2}(s) d s\left[1+\|X\|_{H^{2}}^{2}\right] \\
& \rightarrow 0 \text {, let } t \rightarrow t_{0} \text { and then } r \rightarrow 1 \text {. }
\end{aligned}
$$

The last step follows by a dominated convergence theorem since $\|\left(S\left(t_{0}-r s\right)-S(t-\right.$ $r s)) \Phi^{r}(s) e_{i}\left\|^{2} \leq 4 M_{T}^{2}\right\| \Phi^{r}(s) e_{i} \|^{2}$ and $\Phi^{r}(t) \in \mathcal{N}_{W}^{2}(0, T), t \in[0, T]$. 
For $0 \leq t_{0}<t \leq T$, we can get the same conclusion. Therefore by Theorem [11] we know

$$
\int_{0}^{t} S(t-s) Q(X(s)) d W(s)
$$

has a predictable version for all $t \in[0, T]$. Furthermore, by (A.5), we have

$$
\begin{aligned}
& \left(E\left(\left\|\int_{0}^{t} S(t-s) Q(X(s)) d W(s)\right\|^{2}\right)\right)^{1 / 2} \\
& =\left(\int_{0}^{t}\left(E\left(\|S(t-s) Q(X(s))\|_{L_{2}}^{2}\right)\right) d s\right)^{1 / 2} \\
& \leq\left(\int_{0}^{T} M^{2}(s) d s\right)^{1 / 2}\left(1+\|X\|_{H^{2}}\right) .
\end{aligned}
$$

So far we have proved $\int_{0}^{t} S(t-s) Q(X(s)) d W(s)$ has a predictable version which is an element in $\mathcal{H}^{2}(T, H)$.

4. There is a version of $\int_{0}^{t} \int_{Z} S(t-s) F(X(s-), z) \tilde{N}(d s, d z), t \in[0, T]$, which is in $\mathcal{H}^{2}(T, H)$. It is easy to show $1_{[0, t]}(s) S(t-s) F(X(s-), z)$ is predictable. By (A.6), we have

$$
\begin{aligned}
& \left(E\left\|\int_{0}^{t} \int_{Z} S(t-s) F(X(s-), z) \tilde{N}(d s, d z)\right\|^{2}\right)^{1 / 2} \\
& =\left\{E\left(\int_{0}^{t} \int_{Z} 1_{[0, t]}(s)\|S(t-s) F(X(s), z)\|^{2} \lambda(d z) d s\right)\right\}^{1 / 2} \\
& \quad \leq C^{\frac{1}{2}} M(T)\left\{E\left(\int_{0}^{t}\left(1+\|X(s)\|^{2}\right) d s\right)\right\}^{1 / 2} \\
& \leq C^{\frac{1}{2}} \operatorname{TM}(T)\left(1+\|X\|_{H^{2}}\right) .
\end{aligned}
$$

Letting $t<t_{0}$, we have

$$
\begin{aligned}
&\left\{E\left\|\int_{0}^{t} \int_{Z} S(t-s) F(X(s-), z) \tilde{N}(d s, d z)-\int_{0}^{t_{0}} \int_{Z} S\left(t_{0}-s\right) F(X(s-), z) \widetilde{N}(d s, d z)\right\|^{2}\right\}^{1 / 2} \\
& \leq\left\{E\left(\left\|\int_{0}^{t} \int_{Z}\left(S(t-s)-S\left(t_{0}-s\right)\right) F(X(s-), z) \tilde{N}(d s, d z)\right\|^{2}\right)\right\}^{1 / 2} \\
&+\left\{E\left(\left\|\int_{t}^{t_{0}} \int_{Z} S\left(t_{0}-s\right) F(X(s-), z) \tilde{N}(d s, d z)\right\|^{2}\right)\right\}^{1 / 2} \\
&=\left\{E \int_{0}^{t} \int_{Z}\left\|\left(S(t-s)-S\left(t_{0}-s\right)\right) F(X(s), z)\right\|^{2} \lambda(d z) d s\right\}^{1 / 2} \\
&+\left\{E \int_{t}^{t_{0}} \int_{Z}\left\|S\left(t_{0}-s\right) F(X(s), z)\right\|^{2} \lambda(d z) d s\right\}^{1 / 2} \\
& \leq\left\{E \int_{0}^{t / r} \int_{Z}\left\|\left(S(t-s)-S\left(t_{0}-s\right)\right) F(X(s), z)\right\|^{2} \lambda(d z) d s\right\}^{1 / 2} \\
&+\left\{E \int_{t / r}^{t} \int_{Z}\left\|\left(S(t-s)-S\left(t_{0}-s\right)\right) F(X(s), z)\right\|^{2} \lambda(d z) d s\right\}^{1 / 2}
\end{aligned}
$$




$$
\begin{aligned}
& +C^{\frac{1}{2}} M(T)\left\{\int_{t}^{t_{0}}\left(1+E\left(\|X(s)\|^{2}\right)\right) d s\right\}^{1 / 2} \\
\leq & \left\{E \int_{0}^{t / r} \int_{Z}\left\|\left(S(t-s)-S\left(t_{0}-s\right)\right) F(X(s), z)\right\|^{2} \lambda(d z) d s\right\}^{1 / 2} \\
& +2 C^{\frac{1}{2}} M(T)\left\{E \int_{t / r}^{t}\left(1+\|X(s)\|^{2}\right) d s\right\}^{1 / 2}+C^{\frac{1}{2}} M(T)\left(t_{0}-t\right)^{\frac{1}{2}}\left(1+\|X\|_{H^{2}}\right) \\
\leq & \left\{E \int_{0}^{t / r} \int_{Z}\left\|\left(S(t-r s)-S\left(t_{0}-r s\right)\right) S((r-1) s) F(X(s), z)\right\|^{2} \lambda(d z) d s\right\}^{1 / 2} \\
& +2 C^{\frac{1}{2}} M(T)\left\{E \int_{t / r}^{t}\left(1+\|X(s)\|^{2}\right) d s\right\}^{1 / 2}+C^{\frac{1}{2}} M(T)\left(t_{0}-t\right)^{\frac{1}{2}}\left(1+\|X\|_{H^{2}}\right) .
\end{aligned}
$$

Since

$$
E \int_{0}^{t / r} \int_{Z}\|S((r-1) s) F(X(s), z)\|^{2} \lambda(d z) d s<+\infty
$$

letting $t \rightarrow t_{0}$ and $r \downarrow 1$, by a dominated convergence theorem, we have the following result:

$$
\begin{aligned}
& \int_{0}^{t} \int_{Z} S(t-s) F(X(s-), z) \tilde{N}(d s, d z) \\
& \quad \rightarrow \int_{0}^{t_{0}} \int_{Z} S\left(t_{0}-s\right) F(X(s-), z) \tilde{N}(d s, d z) \quad \text { in } L^{2}(\Omega), \text { as } t \uparrow t_{0}
\end{aligned}
$$

If $t>t_{0}$, similarly we can get

$$
\begin{aligned}
& \int_{0}^{t} \int_{Z} S(t-s) F(X(s-), z) \tilde{N}(d s, d z) \\
& \quad \rightarrow \int_{0}^{t_{0}} \int_{Z} S\left(t_{0}-s\right) F(X(s-), z) \tilde{N}(d s, d z) \quad \text { in } L^{2}(\Omega), \text { as } t \downarrow t_{0} .
\end{aligned}
$$

As $\int_{0}^{t} \int_{Z} S(t-s) F(X(s-), z) \tilde{N}(d s, d z)$ is $\mathcal{F}_{t}$ adapted, thus by Theorem [11] we know

$$
\int_{0}^{t} \int_{Z} S(t-s) F(X(s-), z) \tilde{N}(d s, d z)
$$

has a predictable version which is an element in $\mathcal{H}^{2}(T, H)$.

The third step: We are going to show

$$
\mathcal{F}(\eta, \cdot): \mathcal{H}^{2, \lambda}(T, H) \rightarrow \mathcal{H}^{2, \lambda}(T, H)
$$

is a contraction mapping for all $\eta \in L_{0}^{2}$.

Let $X, \tilde{X} \in \mathcal{H}^{2}(T, H), \eta \in L_{0}^{2}$ and $t \in[0, T]$. Then we have

$$
\begin{aligned}
& \|\mathcal{F}(\eta, X)-\mathcal{F}(\eta, \tilde{X})\|_{L^{2}} \\
& \quad \leq\left\|\int_{0}^{t} S(t-s)[B(X(s))-B(\tilde{X}(s))] d s\right\|_{L^{2}}
\end{aligned}
$$


where \|\|$_{L^{2}}:=\left(E\left(\|\|^{2}\right)\right)^{1 / 2}$. For the first term of the right-hand side of (3.1), we have

$$
\begin{aligned}
& \left\|\int_{0}^{t} S(t-s)[B(X(s))-B(\tilde{X}(s))] d s\right\|_{L^{2}} \\
& \quad=\left\{E\left(\left\|\int_{0}^{t} S(t-s)[B(X(s))-B(\tilde{X}(s))] d s\right\|^{2}\right)\right\}^{1 / 2} \\
& \quad \leq\left\{E\left(\int_{0}^{t}\|S(t-s)[B(X(s))-B(\tilde{X}(s))]\|^{2} d s\right) t\right\}^{1 / 2} \\
& \quad \leq M(T)\left\{E\left(\int_{0}^{t} e^{2 \lambda s} e^{-2 \lambda s}\|X(s)-\tilde{X}(s)\|^{2} d s\right)\right\}^{1 / 2} t^{1 / 2} \\
& \quad \leq M(T)\left(\int_{0}^{t} e^{2 \lambda s} d s\right)^{1 / 2} \sup _{t \in[0, T]} e^{-\lambda t}\left(E\left(\|X(t)-\tilde{X}(t)\|^{2}\right)\right)^{1 / 2} T^{1 / 2} \\
& \quad=T^{1 / 2} M(T) e^{\lambda t}\left(\int_{0}^{t} e^{-2 \lambda s} d s\right)^{1 / 2}\|X-\tilde{X}\|_{2, \lambda, T} .
\end{aligned}
$$

Dividing by $e^{\lambda t}$ both sides of (3.2), we have

$$
\left\|\int_{0}^{t} S(t-s)[B(X(s))-B(\tilde{X}(s))] d s\right\|_{2, \lambda, T} \leq T^{1 / 2} M(T)\left(\int_{0}^{t} e^{-2 \lambda s} d s\right)^{1 / 2}\|X-\tilde{X}\|_{2, \lambda, T} .
$$

Obviously $T^{1 / 2} M(T)\left(\int_{0}^{t} e^{-2 \lambda s} d s\right)^{1 / 2} \rightarrow 0$ as $\lambda \rightarrow \infty$.

For the second term of (3.1), by the Burkholder-Davis-Gundy inequality and (A.5), we have

$$
\begin{aligned}
& \left\|\int_{0}^{t} S(t-s)[Q(X(s))-Q(\tilde{X}(s))] d W(s)\right\|_{L^{2}} \\
& \quad=\left(E\left(\left\|\int_{0}^{t} S(t-s)[Q(X(s))-Q(\tilde{X}(s))] d W(s)\right\|^{2}\right)\right)^{1 / 2} \\
& \quad \leq\left(\int_{0}^{t}\left(E\left(\|S(t-s)[Q(X(s))-Q(\tilde{X}(s))]\|_{L^{2}}^{2}\right)\right) d s\right)^{1 / 2} \\
& \quad \leq\left(\int_{0}^{t} M^{2}(t-s) e^{2 \lambda s} e^{-2 \lambda s} E\|X(s)-\tilde{X}(s)\|_{L^{2}}^{2} d s\right)^{1 / 2} \\
& \quad \leq e^{\lambda t}\left(\int_{0}^{T} M^{2}(s) e^{-2 \lambda s} d s\right)^{1 / 2}\|X-\tilde{X}\|_{2, \lambda, T} .
\end{aligned}
$$

Dividing by $e^{\lambda t}$ both sides of (3.3), we have

$$
\left\|\int_{0}^{t} S(t-s)[Q(X(s))-Q(\tilde{X}(s))] d W(s)\right\|_{2, \lambda, T} \leq\left(\int_{0}^{T} M^{2}(s) e^{-2 \lambda s} d s\right)^{1 / 2}\|X-\tilde{X}\|_{2, \lambda, T} .
$$


Obviously,

$$
\int_{0}^{T} M^{2}(s) e^{-2 \lambda s} d s \rightarrow 0, \quad \text { as } \lambda \rightarrow+\infty
$$

By the Burkholder-Davis-Gundy inequality and (A.6), we have the following estimate for the third term of the right-hand side of (3.1):

$$
\begin{aligned}
\{E & \left.\left(\left\|\int_{0}^{t} \int_{Z} S(t-s)(F(X(s-), z)-F(\tilde{X}(s-), z)) \tilde{N}(d s, d z)\right\|^{2}\right)\right\}^{1 / 2} \\
& \leq\left\{E\left(\int_{0}^{t} \int_{Z}\|S(t-s)(F(X(s), z)-F(\tilde{X}(s), z))\|^{2} \lambda(d z) d s\right)\right\}^{1 / 2} \\
& \leq C^{\frac{1}{2}} M(T)\left\{E\left(\int_{0}^{t}\|X(s)-\tilde{X}(s)\|^{2} d s\right)\right\}^{1 / 2} \\
& \leq C^{\frac{1}{2}} M(T)\left\{\int_{0}^{t} e^{2 \lambda s} e^{-2 \lambda s} E\left(\|X(s)-\tilde{X}(s)\|^{2}\right) d s\right\}^{1 / 2} \\
& \leq C^{\frac{1}{2}} M(T)\left\{\int_{0}^{t} e^{2 \lambda s} d s\right\}^{1 / 2}\left(\sup _{t \in[0, T]} e^{-\lambda t}\left(E\left(\|X(t)-\tilde{X}(t)\|^{2}\right)\right)^{1 / 2}\right) \\
& =C^{\frac{1}{2}} M(T) e^{\lambda t}\left\{\int_{0}^{t} e^{-2 \lambda s} d s\right\}^{1 / 2}\|X-\tilde{X}\|_{2, \lambda, T} .
\end{aligned}
$$

Thus we have

$$
\begin{aligned}
& \left\|\int_{0}^{t} \int_{Z} S(t-s)(F(X(s-), z)-F(\tilde{X}(s-), z)) \tilde{N}(d s, d z)\right\|_{2, \lambda, T} \\
& \quad \leq C^{\frac{1}{2}} M(T)\left\{\int_{0}^{t} e^{-2 \lambda s} d s\right\}^{1 / 2}\|X-\tilde{X}(s)\|_{2, \lambda, T^{*}}
\end{aligned}
$$

Obviously,

$$
\int_{0}^{t} e^{-2 \lambda s} d s \rightarrow 0, \quad \text { as } \lambda \rightarrow+\infty \text {. }
$$

Therefore we have finally proved that there exists an $a(\lambda)<1$ with

$$
\|\mathcal{F}(\eta, X)-\mathcal{F}(\eta, \tilde{X}(s))\|_{2, \lambda, T} \leq a(\lambda)\|X-\tilde{X}\|_{2, \lambda, T}
$$

So, there exists a unique

$$
X: L_{0}^{2} \rightarrow \mathcal{H}^{2}(T, H)
$$

satisfying

$$
X(\eta)=\mathcal{F}(\eta, X(\eta))
$$

which is the unique solution of (2.1). 
The fourth step : We will show the Lipschitz continuity of $X: L_{0}^{2} \rightarrow \mathcal{H}^{2}(T, H)$. By contraction theorem (iii), we only have to prove that the mapping

$$
\mathcal{F}(\cdot, Y): L_{0}^{2} \rightarrow \mathcal{H}^{2}(T, H)
$$

is Lipschitz continuous for all $Y \in \mathcal{H}^{2}(T, H)$, where the Lipschitz constant does not depend on $Y$.

But this is obvious for all $\xi_{1}, \xi_{2} \in L_{0}^{2}$ and $Y \in \mathcal{H}^{2}(T, H)$, as

$$
\left\|\mathcal{F}\left(\xi_{1}, Y\right)-\mathcal{F}\left(\xi_{2}, Y\right)\right\|_{H^{2}}=\left\|S(\cdot)\left(\xi_{1}-\xi_{2}\right)\right\|_{H^{2}} \leq M_{T}\left\|\xi_{1}-\xi_{2}\right\|_{L^{2}}
$$

Before giving the regularity of the mild solution of (2.1), we first need a stochastic Fubini theorem with respect to compensated Poisson measure. Set $\Omega_{T} \times Z=[0, T] \times \Omega \times Z$, let $\mathcal{Z}$ be $\sigma$-algebra generated by open subsets in $Z, \mathcal{P}_{T}$ be the predictable $\sigma$-algebra of $[0, T] \times \Omega$, $d s \otimes P \otimes \lambda(d z)$ be the product of the Lebesgue measure, $P$ and $\lambda(d z)$ on $[0, T] \times \Omega \times Z$. $L^{2}\left(\Omega_{T} \times Z\right):=L^{2}(([0, T] \times \Omega \times Z, d s \otimes P \otimes \lambda(d z)) ; H)$.

Proposition 3.1 Let $(Y, \mathcal{Y}, \mu)$ be a finite measure space and let $\psi: \Omega_{T} \times Y \times Z \rightarrow H$ be a $\mathcal{P}_{T} \otimes \mathcal{Y} \otimes \mathcal{Z}$-measurable mapping such that

$$
\int_{Y}\|\psi(y)\|_{L^{2}\left(\Omega_{T} \times Z\right)} d \mu<\infty .
$$

Then

(1) the process indexed by $t \in[0, T]$

$$
\int_{Y} \psi(y) d \mu
$$

is progressively measurable and belongs to $L^{2}\left(\Omega_{T} \times Z\right)$,

(2) the process indexed by $y \in Y$

$$
\int_{0}^{T} \int_{Z} \psi(s-, z) \tilde{N}(d s, d z)
$$

has an $\mathcal{F}_{T} \otimes \mathcal{Y}$-measurable version $m: \Omega \times Y \rightarrow H$ such that

$$
P\left[m(y)=\int_{0}^{T} \int_{Z} \psi(y) \tilde{N}(d s, d z)\right]=1,
$$

(3) we have

$$
P\left[\int_{Y} m(y) d \mu=\int_{0}^{T} \int_{Z}\left(\int_{Y} \psi(y) d \mu\right) \tilde{N}(d s, d z)\right]=1 .
$$

Proof (1) follows from the following inequality: Let $f$ be a nonnegative $\mathcal{P}_{T} \otimes \mathcal{Y} \otimes \mathcal{Z}$ measurable function. Then

$$
\sqrt{\int_{\Omega_{T} \times Z}\left(\int_{Y} f d \mu\right)^{2} d s \times P \times \lambda(d z)} \leq \int_{Y}\|f\|_{L^{2}\left(\Omega_{T} \times Z, R\right)} d \mu
$$


because

$$
\left(\int_{Y} f(a, y) d u\right)^{2}=\int_{Y \times Y} f\left(a, y_{1}\right) f\left(a, y_{2}\right) d \mu d \mu
$$

and we get (3.4) by the Schwarz inequality. Now suppose that $m$ in (2) exists. Then, by taking $\Omega$ instead of $\Omega_{T} \times Z$ in (3.4), we get

$$
\sqrt{E\left(\int_{Y}\|m(y)\| d \mu\right)^{2}} \leq \int_{Y} \sqrt{E\|m(y)\|^{2}} d \mu \leq(\mu(Y))^{1 / 2}\|\psi(y)\|_{L^{2}\left(\Omega_{T} \times Y \times Z\right)}
$$

by the Burkholder inequality. Hence

$$
\int_{Y} m(y) d \mu
$$

is defined $P$-almost everywhere. Now take $\psi_{n}$ satisfying the assumption of the proposition such that the sequence of the integrals

$$
\int_{Y}\left\|\psi_{n}(y)-\psi(y)\right\|_{L^{2}\left(\Omega_{T} \times Z\right)} d \mu
$$

converges to zero. Then there exists a subsequence $\left(n_{k}: k \in N\right)$ such that:

(a) $\int_{0}^{T} \int_{Z} \psi_{n_{k}}(y) \tilde{N}(d s, d z) \rightarrow \int_{0}^{T} \int_{Z} \psi(y) \tilde{N}(d s, d z)$ in $L^{1}(\Omega ; H)$ for $\mu$-almost all $y \in Y$.

(b) $\int_{0}^{T} \int_{Z}\left(\int_{Y} \psi_{n_{k}}(y) d \mu\right) \tilde{N}(d s, d z) \rightarrow \int_{0}^{T} \int_{Z}\left(\int_{Y} \psi(y) d \mu\right) \tilde{N}(d s, d z)$ in $L^{2}(\Omega ; H)$ for $\mu$-almost all $y \in Y$.

Let us introduce the set $\mathcal{D}$ of all $\mathcal{P}_{T} \otimes \mathcal{Y} \otimes \mathcal{Z}$-measurable processes $\psi$ with

$$
\int_{Y}\|\psi(y)\|_{L^{2}\left(\Omega_{T} \times Z\right)} d \mu<\infty
$$

such that there exists an $m$ satisfying (2) and (3). It is easy to see that $\mathcal{D}$ is a linear space and if we can find $\psi_{n} \in \mathcal{D}$ such that

$$
\int_{Y}\left\|\psi_{n}(y)-\psi(y)\right\|_{L^{2}\left(\Omega_{T} \times Z\right)} d \mu \rightarrow 0
$$

then we would finish the proof. Indeed, take the corresponding functions $m_{n}$. Then the sequence $\left(m_{n}: n \in N\right)$ is Cauchy in $L^{1}(\Omega \times Y ; H)$ due to (3.5) and $\psi$ belongs to $\mathcal{D}$ due to (a) and (b). Now we will show how to construct the approximating sequence $\psi_{n}$. We assume that $\lambda(d z)$ is finite. By Lemma A.1.4 in [11] we can find mappings $F_{n}$ on $H$. The simple functions $F_{n} \psi$ take values in the finite dimensional subspace of $H$. Moreover,

$$
\int_{Y}\left\|F_{n} \psi(y)-\psi(y)\right\|_{L^{2}\left(\Omega_{T} \times Z\right)} d \mu \downarrow 0,
$$

and if $F_{n} \psi \in \mathcal{D}, n \in N$, then $\psi \in \mathcal{D}$. Now to show that $F_{n} \psi \in \mathcal{D}$, we will take advantage of the fact that each $F_{n} \psi$ is bounded in $H$ and

$$
\int_{Y}\left\|\phi_{n}(y)-\phi(y)\right\|_{L^{2}\left(\Omega_{T} \times Z\right)} d \mu \rightarrow 0
$$


if and only if

$$
\int_{Y}\left\|\phi_{n}(y)-\phi(y)\right\|_{L^{1}\left(\Omega_{T} \times Y \times Z\right)} d \mu \rightarrow 0
$$

for $\phi_{n}$ uniformly bounded in $H$. So as $F_{n}$ is of the form

$$
\sum_{k=1}^{m} I_{C_{k}} B_{k}
$$

where $\left(C_{k}: k \leq m\right)$ is a $\mathcal{P}_{T} \otimes \mathcal{Y} \otimes \mathcal{Z}$-decomposition of $\Omega_{T} \times Y \times Z$ and $B_{k}, k \leq m$ are elements in the finite dimensional subspace of $H$. We conclude that $F_{n} \psi \in \mathcal{D}$ provided $I_{C_{k}} B_{k} \in \mathcal{D}$ due to linearity of $\mathcal{D}$. Another reduction shows that this is true if

$$
I_{C_{k}^{1} \times C_{k}^{2} \times C_{k}^{3} B_{k}} \in \mathcal{D}
$$

for every $C_{k}^{1} \in \mathcal{P}_{T}, C_{k}^{2} \in \mathcal{Y}, C_{k}^{3} \in \mathcal{Z}$ as $I_{C_{k}}$ can be approximated by $I_{C_{k}^{0}}$ in $L^{1}\left(\Omega_{T} \times Y \times Z\right)$ where $C_{k}^{0}$ is a disjoint union of sets of the type $C_{k}^{1} \times C_{k}^{2} \times C_{k}^{3}$. Finally, as $I_{C_{k}^{1}}$ is a predictable process, it can be approximated by simple bounded real processes in $L^{1}\left(\Omega_{T}\right)$. So, we will finish the proof by showing that

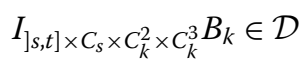

for $s<t, C_{s} \in \mathcal{F}_{s}$, but this is obvious. When $\lambda(d z)$ is $\sigma$-finite, there exists a sequence $A_{n}$ satisfying $\lambda\left(A_{n}\right)<+\infty$ and $A_{n} \uparrow Z$. Obviously,

$$
\psi(y) I_{A_{n}} \in \mathcal{D} \quad \text { and } \quad \int_{Z}\left\|\psi I_{A_{n}}(z)-\psi(z)\right\|_{L^{2}\left(\Omega_{T} \times Z\right)} d \mu \rightarrow 0
$$

therefore $\psi \in \mathcal{D}$.

In the following we give a type of the Burkholder-Davis-Gundy inequality which will play an important role in proving the regularity property of the mild solution of (2.1).

Lemma 3.1 If $A$ is the infinitesimal generator of pseudo-contraction $C_{0}$-semigroup $S(t)$, $t \leq T$, and $g(t, z)$ is an $H$-valued progressively measurable mapping with respect to $\left\{\mathcal{F}_{t}\right\}_{t \geq 0}$ such that

$$
\int_{0}^{T} \int_{Z}\|g(t, z)\|^{2} \lambda(d z) d t<\infty, \quad \text { a.s. }
$$

then for any $t \in[0, T]$,

$$
E\left(\sup _{0 \leq s \leq t}\left\|\int_{0}^{s} \int_{Z} S(s-u) g(u-, z) \tilde{N}(d u, d z)\right\|^{2}\right) \leq 8 e^{4 r t} E \int_{0}^{t} \int_{Z}\|g(s, z)\|^{2} \lambda(d z) d s
$$

for some number $r \geq 0$. 
Proof Let us define

$$
Y(t)=\int_{0}^{t} \int_{Z} S(t-s) g(s-, z) \tilde{N}(d s, d z) .
$$

Assume that $g(t, z)$ takes values in $\mathcal{D}(A)$ and

$$
E \int_{0}^{t} \int_{Z}\|g(s, z)\|^{2} \lambda(d z) d s+E \int_{0}^{t} \int_{Z}\|A g(s, z)\|^{2} \lambda(d z) d s<+\infty \quad \text { for all } t>0 .
$$

Since $A$ is the infinitesimal generator of a $C_{0}$-semigroup, $A$ is closed. And by (3.6) we can check that

$$
\int_{0}^{t} \int_{Z} S(t-s) g(s-, z) \tilde{N}(d s, d z) \in \mathcal{D}(A)
$$

for all $t \geq 0$ and

$$
A \int_{0}^{t} \int_{Z} S(t-s) g(s-, z) \tilde{N}(d s, d z)=\int_{0}^{t} \int_{Z} A S(t-s) g(s-, z) \tilde{N}(d s, d z) .
$$

By (3.6) and Proposition 3.1 with $Y$ replaced with $[0, T]$, we have

$$
\begin{aligned}
\int_{0}^{t} A Y(s) d s & =\int_{0}^{T} \int_{0}^{T} \int_{Z} 1_{[0, t]}(s) 1_{[0, s]}(u) A S(s-u) g(u-, z) \tilde{N}(d u, d z) d s \\
& =\int_{0}^{T} \int_{Z} \int_{0}^{T} 1_{[u, t]}(s) 1_{[0, t]}(u) A S(s-u) g(u-, z) d s \tilde{N}(d u, d z) \\
& =\int_{0}^{t} \int_{Z} S(t-u) g(u-, z) \tilde{N}(d u, d z)-\int_{0}^{t} \int_{Z} g(u-, z) \tilde{N}(d u, d z) \\
& =Y(t)-\int_{0}^{t} \int_{Z} g(u-, z) \tilde{N}(d u, d z) .
\end{aligned}
$$

By Itô’s formula, we get

$$
\begin{aligned}
\|Y(t)\|^{2}= & 2 \int_{0}^{t}\langle A Y(s), Y(s)\rangle d s+\int_{0}^{t} \int_{Z}\|g(s, z)\|^{2} \lambda(d z) d s \\
& +\int_{0}^{t} \int_{Z}\left(2\langle Y(s-), g(s-, z)\rangle+\|g(s-, z)\|^{2}\right) \tilde{N}(d s, d z) .
\end{aligned}
$$

Set

$$
\tilde{Y}(t)=\sup _{0 \leq s \leq t}\|Y(s)\| \quad \text { and } \quad \tilde{g}(t)=\int_{0}^{t} \int_{Z}\|g(s, z)\|^{2} \lambda(d z) d s .
$$

So, we have

$$
\begin{aligned}
(\tilde{Y}(t))^{2} \leq & \sup _{0 \leq s \leq t} 2 \int_{0}^{s}\langle A Y(u), Y(u)\rangle d u+\int_{0}^{t} \int_{Z}\|g(u, z)\|^{2} \lambda(d z) d u \\
& +\sup _{0 \leq s \leq t} \int_{0}^{s} \int_{Z}\left(2\langle Y(u-), g(u-, z)\rangle+\|g(u-, z)\|^{2}\right) \tilde{N}(d u, d z) .
\end{aligned}
$$


Since $A$ is pseudo-contraction, there exists $r \geq 0$ such that

$$
\langle A x, x\rangle \leq r\|x\|^{2} \text { for all } x \in \mathcal{D}(A) .
$$

Define $T_{n}:=\inf \{t \mid\|Y(t)\|>n\}$. Then by (3.7), (3.8) and B-D-G inequality, we can get the following estimate:

$$
\begin{aligned}
E\left(\tilde{Y}\left(t \wedge T_{n}\right)\right)^{2} \leq & 2 r E \int_{0}^{t \wedge T_{n}}\|Y(s)\|^{2} d s+E \tilde{g}\left(t \wedge T_{n}\right) \\
& +2 E \sup _{0 \leq s \leq t \wedge T_{n}} \int_{0}^{s} \int_{Z}\langle Y(u-), g(u-, z)| \tilde{N}(d u, d z) \\
& +E \sup _{0 \leq s \leq t \wedge T_{n}} \int_{0}^{s} \int_{Z}\|g(u-, z)\|^{2} \tilde{N}(d u, d z) \\
\leq & 2 r E \int_{0}^{t \wedge T_{n}}\|Y(s)\|^{2} d s+E \tilde{g}\left(t \wedge T_{n}\right) \\
& +2 E\left(\int_{0}^{t \wedge T_{n}} \int_{Z}\left\langle Y(s-),\left.g(s-, z)\right|^{2} N(d s, d z)\right)^{1 / 2}\right. \\
& +E\left(\int_{0}^{t \wedge T_{n}} \int_{Z}\|g(s-, z)\|^{4} N(d s, d z)\right)^{1 / 2} \\
\leq & 2 r E \int_{0}^{t \wedge T_{n}}\|Y(s)\|^{2} d s+E \tilde{g}\left(t \wedge T_{n}\right) \\
& +2 E\left(\int_{0}^{t \wedge T_{n}} \int_{Z}\left\langle Y(s-),\left.g(s-, z)\right|^{2} N(d s, d z)\right)^{1 / 2}\right. \\
& +E\left(\int_{0}^{t \wedge T_{n}} \int_{Z}\|g(s-, z)\|^{2} N(d s, d z)\right) \\
\leq & 2 r E \int_{0}^{t \wedge T_{n}}\|Y(s)\|^{2} d s+2 E \tilde{g}\left(t \wedge T_{n}\right)+\frac{1}{2} E\left(\tilde{Y}\left(t \wedge T_{n}\right)\right)^{2}+2 E \tilde{g}\left(t \wedge T_{n}\right) . \\
& 2 r E \int_{0}^{t \wedge T_{n}}\|Y(s)\|^{2} d s+2 E \tilde{g}\left(t \wedge T_{n}\right) \\
& +2 E\left(\int_{0}^{t \wedge T_{n}} \int_{Z}\|Y(s-)\|^{2}\|g(s-, z)\|^{2} N(d s, d z)\right)^{1 / 2} \| Y T_{n} \\
& +2 E\left(\int _ { 0 } ^ { t \wedge T _ { n } } \int _ { Z } \left\langleY(s-), g(s-, z)\left\|^{2} N(d z, d s)\right\|^{2} d s+2 E \tilde{g}\left(t \wedge T_{n}\right)\right.\right. \\
\leq & 2 r E \int_{0}^{t \wedge T_{n}}\|Y(s)\|^{2} d s+2 E \tilde{g}\left(t \wedge T_{n}\right) \\
& \left.2 r\left(t \wedge T_{n}\right)\left(\int_{0}^{t \wedge T_{n}} \int\|g(s-, z)\|^{2} N(d s, d z)\right)^{1 / 2}\right] \\
& \\
&
\end{aligned}
$$

So, by Gronwall inequality, we have

$$
E\left(\tilde{Y}\left(t \wedge T_{n}\right)\right)^{2} \leq 8 e^{4 r t} E \int_{0}^{t \wedge T_{n}} \int_{Z}\|g(s, z)\|^{2} \lambda(d z) d s .
$$


By the Fatou lemma, we have

$$
E(\tilde{Y}(t))^{2} \leq 8 e^{4 r t} E \int_{0}^{t} \int_{Z}\|g(s, z)\|^{2} \lambda(d z) d s .
$$

Without assuming $g(t, z)$ takes values in $\mathcal{D}(A)$, we define $g_{n}(t, z)=n R(n, A) g(t, z)$ where $R(n, A), n \in \mathbb{N}$, is the resolvent of $A$, then we know $g_{n}(t, z)$ takes values in $\mathcal{D}(A)$ and satisfies (3.6) under the following condition:

$$
E \int_{0}^{t} \int_{Z}\|g(s, z)\|^{2} \lambda(d z) d s<+\infty
$$

Define

$$
Y_{n}(t)=\int_{0}^{t} \int_{Z} S(t-s) g_{n}(s-, z) \tilde{N}(d s, d z), \quad \tilde{Y}_{n}(t)=\sup _{0 \leq s \leq t}\left\|Y_{n}(t)\right\| .
$$

Then instead $\tilde{Y}(t), \tilde{Y}_{n}(t)$ satisfies (3.9). By the Burkholder-Davis-Gundy inequality and dominated convergence theorem, we have

$$
E \int_{0}^{t} \int_{Z}\left\|g_{n}(s, z)-g(s, z)\right\|^{2} \lambda(d z) d s \rightarrow 0, \quad \text { as } n \rightarrow+\infty .
$$

Then it is easy to check

$$
E\left(\sup _{0 \leq s \leq t}\left\|Y_{n}(s)-Y(s)\right\|^{2}\right) \rightarrow 0, \quad \text { as } n \rightarrow+\infty .
$$

So, under condition (3.10), (3.9) also follows without assumption that $g(t, z)$ takes values in $\mathcal{D}(A)$. In order to relax (3.10), we define stopping times

$$
\tau_{n}:=\inf \left\{t \mid \int_{0}^{t} \int_{Z}\|g(s, z)\|^{2} \lambda(d z) d s \geq n\right\} .
$$

Then

$$
\begin{gathered}
E\left(\sup _{\left\{0 \leq s \leq t \wedge \tau_{n}\right\}}\left\|\int_{0}^{s} \int_{Z} S(s-u) g(u-, z)\right\|^{2} \tilde{N}(d u, d z)\right) \\
\quad \leq 8 e^{4 r t} E\left(\int_{0}^{t \wedge \tau_{n}} \int_{Z}\|g(s, z)\|^{2} \lambda(d z) d s\right) \\
\quad \leq 8 e^{4 r t} E\left(\int_{0}^{t} \int_{Z}\|g(s, z)\|^{2} \lambda(d z) d s\right) .
\end{gathered}
$$

Therefore by Fatou lemma, the assertion follows.

In order to study the regularity property of the mild solution of (2.1), we introduce an approximation system of (2.1) in the following:

$$
\begin{aligned}
X(t)= & R(l) \eta+\int_{0}^{t}[A X(s) d s+R(l) B(X(s))] d s+\int_{0}^{t} R(l) Q(X(s)) d W(s) \\
& +\int_{0}^{t} \int_{Z} R(l) F(X(s-), z) \widetilde{N}(d s, d z),
\end{aligned}
$$


where $l \in \rho(A)$ which is the resolvent set of $A$, and $R(l):=l R(l, A), R(l, A)$ is the resolvent of $A$. We say a stochastic process is $R C L L$ if each of it's sample path is right continuous with left limit. So a stochastic process $X(t)$ is called a strong solution of (2.1), if it is $R C L L$, $\mathcal{F}_{t}$-adapted, $X(t) \in \mathcal{D}(A)$ and satisfies $(2.1)$.

Theorem 3.2 Let $\eta \in L^{2}(\Omega, H)$ and $\mathcal{F}_{0}$-measurable. In addition to assumptions in Theorem 3.1 we assume $A$ is dissipative with $x \in H$. Then the mild solution of (2.1) is RCLL.

Proof Let $l \in \rho(A)$, obviously $A R(l)=A l R(l, A)=l-l^{2} R(l, A)$ are bounded operators. So (3.11) has unique strong solution. In fact by Theorem 3.1 we know (3.11) has a unique mild solution denoted by $X^{l}(t)$ and the following hold:

$$
\begin{aligned}
& \int_{0}^{T} \int_{0}^{t}\left\|A S(t-s) R(l) B\left(X^{l}(s)\right)\right\| d s d t<\infty, \\
& E\left(\int_{0}^{T} \int_{0}^{t}\left\|A S(t-s) R(l) Q\left(X^{l}(s)\right)\right\|_{L_{2}^{0}}^{2} d s d t\right)<\infty, \\
& E\left(\int_{0}^{T} \int_{0}^{t} \int_{Z}\left\|A S(t-s) R(l) F\left(X^{l}(s), z\right)\right\|^{2} \lambda(d z) d s d t\right)<\infty .
\end{aligned}
$$

Thus by Fubini theorem, we have

$$
\begin{aligned}
\int_{0}^{t} & \int_{0}^{s} A S(u-s) R(l) B\left(X^{l}(s)\right) d s d u \\
\quad & \int_{0}^{t} \int_{s}^{t} A S(u-s) R(l) B\left(X^{l}(s)\right) d u d s \\
\quad= & \int_{0}^{t} S(t-s) R(l) B\left(X^{l}(s)\right) d s-\int_{0}^{t} R(l) B\left(X^{l}(s)\right) d s .
\end{aligned}
$$

On the other hand, by the stochastic Fubini theorem for Q-Wiener processes in [11], we have

$$
\begin{aligned}
\int_{0}^{t} & \int_{0}^{u} A S(u-s) R(l) Q\left(X^{l}(s)\right) d W(s) d u \\
\quad & \int_{0}^{t} \int_{s}^{t} A S(u-s) R(l) Q\left(X^{l}(s)\right) d u d W(s) \\
\quad= & \int_{0}^{t} S(t-s) R(l) Q\left(X^{l}(s)\right) d W(s)-\int_{0}^{t} R(l) Q\left(X^{l}(s)\right) d W(s) .
\end{aligned}
$$

And by Proposition 3.1

$$
\begin{aligned}
\int_{0}^{t} & \int_{0}^{u} \int_{Z} A S(u-s) R(l) F\left(X^{l}(s-), z\right) \tilde{N}(d s, d z) d u \\
\quad= & \int_{0}^{t} \int_{Z} \int_{s}^{t} A S(u-s) R(l) F\left(X^{l}(s-), z\right) d u \tilde{N}(d s, d z) \\
\quad= & \int_{0}^{t} \int_{Z} S(t-s) R(l) F\left(X^{l}(s-), z\right) \tilde{N}(d s, d z)-\int_{0}^{t} \int_{Z} R(l) F\left(X^{l}(s-), z\right) \widetilde{N}(d s, d z) .
\end{aligned}
$$


Hence, $A X^{l}(t)$ is integrable almost surely and

$$
\begin{aligned}
\int_{0}^{t} A X^{l}(s) d s= & S(t) R(l) \eta-R(l) \eta+\int_{0}^{t} S(t-s) R(l) B\left(X^{l}(s)\right) d s-\int_{0}^{t} R(l) B\left(X^{l}(s)\right) d s \\
& +\int_{0}^{t} S(t-s) R(l) Q\left(X^{l}(s)\right) d W(s)-\int_{0}^{t} R(l) Q\left(X^{l}(s)\right) d W(s) \\
& +\int_{0}^{t} \int_{Z} S(t-s) R(l) F\left(X^{l}(s-), z\right) \tilde{N}(d s, d z) \\
& -\int_{0}^{t} \int_{Z} R(l) F\left(X^{l}(s-), z\right) \tilde{N}(d s, d z) \\
= & X^{l}(t)-R(l) \eta-\int_{0}^{t} R(l) B\left(X^{l}(s)\right) d s-\int_{0}^{t} R(l) Q\left(X^{l}(s)\right) d W(s) \\
& -\int_{0}^{t} \int_{Z} R(l) F\left(X^{l}(s-), z\right) \tilde{N}(d s, d z) .
\end{aligned}
$$

So far, we prove $X^{l}(t) \in \mathcal{D}(A), t \in[0, T]$, is the unique strong solution of (3.11). Let $X(t)$ be the mild solution of (2.1). Then we consider

$$
\begin{aligned}
X(t)-X^{l}(t)= & S(t)(\eta-R(l) \eta) \\
& +\int_{0}^{t} S(t-s)\left[B(X(s))-R(l) B\left(X^{l}(s)\right)\right] d s \\
& +\int_{0}^{t} S(t-s)\left[Q(X(s))-R(l) Q\left(X^{l}(s)\right)\right] d W(s) \\
& +\int_{0}^{t} \int_{Z} S(t-s)\left[F(X(s-), z)-R(l) F\left(X^{l}(s-), z\right)\right] \tilde{N}(d s, d z)
\end{aligned}
$$

for any $t \geq 0$. We have that for any $T \geq 0$,

$$
\begin{aligned}
E \sup _{0 \leq t \leq T}\left\|X(t)-X^{l}(t)\right\|^{2} \\
\leq 16 E \sup _{0 \leq t \leq T}\left\|\int_{0}^{t} S(t-s) R(l)\left[B(X(s))-B\left(X^{l}(s)\right)\right] d s\right\|^{2} \\
\quad+16 E \sup _{0 \leq t \leq T}\left\|\int_{0}^{t} S(t-s) R(l)\left[Q(X(s))-Q\left(X^{l}(s)\right)\right] d W(s)\right\|^{2} \\
\quad+16 E \sup _{0 \leq t \leq T}\left\|\int_{0}^{t} \int_{Z} S(t-s) R(l)\left[F(X(s-), z)-F\left(X^{l}(s-), z\right)\right] \widetilde{N}(d s, d z)\right\|^{2} \\
\quad+16\left\{E \sup _{0 \leq t \leq T} \| S(t)(\eta-R(l) \eta)+\int_{0}^{t} S(t-s)[I-R(l)] B(X(s)) d s\right. \\
\quad+\int_{0}^{t} S(t-s)[I-R(l)] Q(X(s)) d W(s) \\
\left.\quad+\int_{0}^{t} \int_{Z} S(t-s)[I-R(l)] F(X(s-), z) \tilde{N}(d s, d z) \|^{2}\right\} \\
:=16\left[I_{1}+I_{2}+I_{3}+I_{4}\right] .
\end{aligned}
$$


We consider

$$
\begin{aligned}
I_{1} & =E \sup _{0 \leq t \leq T}\left\|\int_{0}^{t} S(t-s) R(l)\left[B(X(s))-B\left(X^{l}(s)\right)\right] d s\right\|^{2} \\
& \leq E \sup _{0 \leq t \leq T}\left(\int_{0}^{t}\left\|R(l) S(t-s)\left[B(X(s))-B\left(X^{l}(s)\right)\right]\right\| d s\right)^{2} .
\end{aligned}
$$

When $l$ is big enough, there exists constant $M$ such that $\|R(l)\| \leq M$, so we have

$$
\begin{aligned}
I_{1} & \leq C^{2} M^{2} M_{T}^{2} E \sup _{0 \leq t \leq T}\left(\int_{0}^{t}\left\|X(s)-X^{l}(s)\right\| d s\right)^{2} \\
& \leq C^{2} M^{2} M_{T}^{2} \int_{0}^{T} E \sup _{0 \leq u \leq s}\left\|X(u)-X^{l}(u)\right\|^{2} d s .
\end{aligned}
$$

Then we consider

$$
\begin{aligned}
I_{2} & :=E \sup _{0 \leq t \leq T}\left\|\int_{0}^{t} S(t-s) R(l)\left[Q(X(s))-Q\left(X^{l}(s)\right)\right] d W(s)\right\|^{2} \\
& =E \sup _{0 \leq t \leq T}\left\|\int_{0}^{t} S(t-s) l R(l, A)\left[Q(X(s))-Q\left(X^{l}(s)\right)\right] d W(s)\right\|^{2} \\
& =E \sup _{0 \leq t \leq T}\left\|\int_{0}^{t} S(t-s) \int_{0}^{+\infty} l e^{-l u} T(u)\left[Q(X(s))-Q\left(X^{l}(s)\right)\right] d u d W(s)\right\|^{2} \\
& \leq E \int_{0}^{T}\left\|\int_{0}^{+\infty} l e^{-l u} T(u)\left[Q(X(s))-Q\left(X^{l}(s)\right)\right] d u\right\|_{L_{2}}^{2} d s \\
& \leq E \int_{0}^{T}\left(\int_{0}^{+\infty} l e^{-l u}\left\|T(u)\left[Q(X(s))-Q\left(X^{l}(s)\right)\right]\right\|_{L_{2}} d u\right)^{2} d s \\
& \leq C(l) E \int_{0}^{T}\left\|X(s)-X^{l}(s)\right\|^{2} d s \leq C(l) E \int_{0}^{T} \sup _{0 \leq u \leq s}\left\|X(u)-X^{l}(u)\right\|^{2} d s,
\end{aligned}
$$

where $C(l) \rightarrow 0$ as $l \rightarrow \infty$. By (A.6) and Lemma 3.1 we have

$$
\begin{aligned}
I_{3} & =E \sup _{0 \leq t \leq T}\left\|\int_{0}^{t} \int_{Z} S(t-s) R(l)\left[F(X(s-), z)-F\left(X^{l}(s-), z\right)\right] \tilde{N}(d s, d z)\right\|^{2} \\
& \leq C(T) E \int_{0}^{T} \int_{Z}\left\|F(X(s), z)-F\left(X^{l}(s), z\right)\right\|^{2} \lambda(d z) d s \\
& \leq C(T) \int_{0}^{T} E \sup _{0 \leq u \leq s}\left\|X(u)-X^{l}(u)\right\|^{2} d s .
\end{aligned}
$$

Finally we consider

$$
\begin{aligned}
I_{4}= & E \sup _{0 \leq t \leq T} \| S(t)(\eta-R(l) \eta)+\int_{0}^{t} S(t-s)[I-R(l)] B(X(s)) d s \\
& +\int_{0}^{t} S(t-s)[I-R(l)] Q(X(s)) d W(s) \\
& +\int_{0}^{t} \int_{Z} S(t-s)[I-R(l)] F(X(s-), z) \tilde{N}(d s, d z) \|^{2} .
\end{aligned}
$$


It is easy to check

$$
E \sup _{0 \leq t \leq T}\|S(t)(\eta-R(l) \eta)\|^{2} \rightarrow 0, \quad \text { as } l \rightarrow \infty
$$

By dominated convergence theorem, we have

$$
\begin{aligned}
E & \sup _{0 \leq t \leq T}\left\|\int_{0}^{t} S(t-s)[I-R(l)] B(X(s)) d s\right\|^{2} \\
& \leq E \sup _{0 \leq t \leq T}\left(\int_{0}^{t}\|[I-R(l)] S(t-s) B(X(s))\| d s\right)^{2} \\
& \leq M_{T}^{2} E\left(\int_{0}^{T}\|[I-R(l)] B(X(s))\| d s\right)^{2} \\
& \leq T M_{T}^{2} \int_{0}^{T} E\|[I-R(l)] B(X(s))\|^{2} d s \rightarrow 0, \quad \text { as } l \rightarrow \infty .
\end{aligned}
$$

By Burkholder-Davis-Gundy inequality, we have

$$
\begin{gathered}
E \sup _{0 \leq t \leq T}\left\|\int_{0}^{t} S(t-s)(I-R(l)) Q(X(s)) d W(s)\right\|^{2} \\
\quad \leq E \int_{0}^{T}\|(I-R(l)) S(t-s) Q(X(s))\|_{L_{2}}^{2} d s \\
\quad \leq \tilde{C} E \int_{0}^{T}\|S(t-s) Q(X(s))\|_{L_{2}}^{2} d s<+\infty
\end{gathered}
$$

for $\tilde{C}>0$. So, by dominated convergence theorem and the fact that

$$
\|(I-R(l)) h\| \rightarrow 0, \quad \text { as } l \rightarrow+\infty
$$

for $h \in H$, we get

$$
(3.12)=E \int_{0}^{T} \sum_{i}\left\|(I-R(l)) S(t-s) Q(X(s)) e_{i}\right\|^{2} d s \rightarrow 0, \quad \text { as } l \rightarrow \infty .
$$

By Lemma 3.1, we get

$$
\begin{aligned}
E & \sup _{0 \leq t \leq T}\left\|\int_{0}^{t} \int_{Z} S(t-s)[I-R(l)] F(X(s-), z) \tilde{N}(d s, d z)\right\|^{2} \\
& \leq M_{T}^{2} E \int_{0}^{T} \int_{Z}\|[I-R(l)] F(X(s), z)\|^{2} \lambda(d z) d s \rightarrow 0, \quad \text { as } l \rightarrow \infty .
\end{aligned}
$$

After discussing about $I_{i}, i=1,2,3$, 4, we can get the following estimate

$$
\begin{aligned}
E & \sup _{0 \leq t \leq T}\left\|X(t)-X^{l}(t)\right\|^{2} \\
& \leq\left[C(T)+C(l)+C^{2} M^{2} M_{T}^{2}\right] \int_{0}^{T} E \sup _{0 \leq u \leq s}\left\|X(u)-X^{l}(u)\right\|^{2} d s+g(l),
\end{aligned}
$$


where $g(l) \rightarrow 0$ as $l \rightarrow \infty$. By Gronwall theorem, we have

$$
E \sup _{0 \leq t \leq T}\left\|X(t)-X^{l}(t)\right\|^{2} \leq g(l) e^{\left[C(T)+C(l)+C^{2} M^{2} M_{T}^{2}\right] T} \rightarrow 0, \quad \text { as } l \rightarrow \infty .
$$

Therefore we know $X(t)$ is $R C L L$ on $[0, T]$ a.s.

\section{Example}

Example 4.1 Consider the following stochastic heat equation

$$
d y(x, t)=\frac{\partial^{2} y(x, t)}{\partial x^{2}} d t+b(x) f(y(\cdot, t)) d B_{t}+\int_{|z| \leq 1} z^{2} x \tilde{N}(d t, d z), \quad t \geq 0,0<x<1
$$

and

$$
y(0, t)=y(1, t)=0, \quad t \geq 0 ; \quad y(x, 0)=y_{0}(x), \quad 0 \leq x \leq 1,
$$

where $B_{t}, t \geq 0$, is a real standard Brownian motion, $\tilde{N}(d t, d z)$ is a Poisson compensated martingale measure with character measure $\lambda(d z)$ on $\mathbb{R}, b \in L^{2}(0,1):=H$ and $f$ is a real Lipschitz continuous function on $H$ satisfying $|f(u)| \leq c\|u\|$ for some $c>0$ and $u \in H$. Let $A=\frac{\partial^{2}}{\partial x^{2}}$ with $\mathcal{D}(A)=H_{0}^{1}(0,1) \cap H^{2}(0,1)$, then we have

$$
\langle u, A u\rangle_{H} \leq-\pi\|u\|^{2} \quad \text { for } u \in \mathcal{D}(A) .
$$

For the sake of simplicity, we assume $\|b\|=1$. It is easy to check that (A.1)-(A.5) are satisfied. So Theorem 3.1 and Theorem 3.2 hold true for (4.1).

Example 4.2 Denote $X(t, x)$ a process satisfying $X(0, x)=x$. Then we consider the semilinear stochastic partial differential equation:

$$
\begin{aligned}
d X(t, x)= & a \frac{\partial^{2}}{\partial x^{2}} X(t, x) d t+[\varphi(t) X(t, x)] d t+b(X(t, x)) d W(t) \\
& +\int_{|z| \leq c} z X(t-, x) \tilde{N}(d z, d t), \\
X(t, 0)= & X(t, \pi)=0 \quad \text { for } t \geq 0, a>0, c>0, x \in H:=L^{2}(0, \pi)
\end{aligned}
$$

for some negative constant $a<0$, and some $M>0, \varphi(\cdot): \mathbb{R}_{+} \rightarrow \mathbb{R}^{1}$ is a bounded function with $|\varphi(t)| \leq M$ for all $t \geq 0, b(x): H \rightarrow \mathbb{R}^{1}$ is nonlinear and Lipschitz continuous with $b(0)=0,|b(x)| \leq M\|x\|, W(t), t \geq 0$, is an $H$-valued $Q$-wiener process with covariance operator $Q, \operatorname{tr} Q<\infty$.

Let $C=\int_{|z| \leq c} z^{2} \lambda(d z)<+\infty$ and $A=a \frac{\partial^{2}}{\partial x^{2}}$ with domain

$$
\mathcal{D}(A)=\left\{h \in H: \frac{\partial h}{\partial x}, \frac{\partial^{2} h}{\partial x^{2}} \in H, h(0)=h(\pi)=0\right\} .
$$

So, it is easy to deduce

$$
\langle A x, x\rangle_{H} \leq a\|x\|^{2}, \quad x \in \mathcal{D}(A)
$$


It is easy to check that (A.1)-(A.5) are satisfied. So, Theorem 3.1 and Theorem 3.2 hold true for (4.3).

\section{Competing interests}

The author declares that he has no competing interests.

\section{Author's contributions}

The author read and approved the final manuscript.

\section{Acknowledgements}

The author is thankful to the referee for careful reading and insightful comments which led to many improvements of the earlier version. The work was supported by Tianyuan Foundation of NSF (Grant No. 11126079), CPSF (Grant No. 2013M530559) and Fundamental Research Funds for the Central Universities (Grant No. CDJRC10100011).

Received: 23 April 2013 Accepted: 28 May 2013 Published: 18 June 2013

\section{References}

1. Applebaum, D: Lévy Processes and Stochastic Calculus. Cambridge University Press, Cambridge (2004)

2. Protter, PE: Stochastic Integration and Differential Equations, 2nd edn. Springer, New York (2004)

3. Peszat, S, Zabczyk, J: Stochastic Partial Differential Equation with Lévy Noise: An Evolution Equation Approach. Cambridge University Press, Cambridge (2007)

4. Albeverio, S, Wu, JL, Zhang, TS: Parabolic SPDES driven by Poisson white noise. Stoch. Process. Appl. 74, 21-36 (1998)

5. Fournier, N: Malliavin calculus for parabolic SPDES with jumps. Stoch. Process. Appl. 87, 115-147 (2000)

6. Mueller, C: The heat equation with Lévy noise. Stoch. Process. Appl. 74, 67-82 (1998)

7. Mytnik, L: Stochastic partial differential equations driven by stable noise. Probab. Theory Relat. Fields 123, 157-201 (2002)

8. Zhou, GL, Hou, ZT: Stochastic generalized porous media equations with Lévy jump. Acta Math. Sin. 27, 1671-1696 (2011)

9. Brzeźniak, Z, Liu, W, Zhu, J: Strong solutions for SPDE with locally monotone coefficients driven by Lévy noise. arXiv:1108.0343

10. Luo, JW, Liu, K: Stability of infinite dimensional stochastic evolution equations with memory and Markovian jumps. Stoch. Process. Appl. 118, 864-895 (2008)

11. Röckner, M: Introduction to Stochastic Partial Differential Equation. Purdue University Press, West Lafayette (2007)

doi:10.1186/1687-1847-2013-175

Cite this article as: Zhou: Global well-posedness of a class of stochastic equations with jumps. Advances in Difference Equations 2013 2013:175.

\section{Submit your manuscript to a SpringerOpen ${ }^{\circ}$ journal and benefit from:}

- Convenient online submission

- Rigorous peer review

- Immediate publication on acceptance

Open access: articles freely available online

- High visibility within the field

- Retaining the copyright to your article 